\title{
LETTER \\ Improvement of Auctioneer's Revenue under Incomplete Information in Cognitive Radio Networks
}

\author{
Jun MA ${ }^{\dagger \mathrm{a})}$, Yonghong ZHANG $^{\dagger \mathrm{b})}$, Nonmembers, and Shengheng LIU ${ }^{\dagger \dagger \mathrm{c})}$, Student Member
}

\begin{abstract}
SUMMARY In this letter, the problem of how to set reserve prices so as to improve the primary user's revenue in the second price-sealed auction under the incomplete information of secondary users' private value functions is investigated. Dirichlet process is used to predict the next highest bid based on historical data of the highest bids. Before the beginning of the next auction round, the primary user can obtain a reserve price by maximizing the additional expected reward. Simulation results show that the proposed scheme can achieve an improvement of the primary user's averaged revenue compared with several counterparts.

key words: cognitive radio networks, second price sealed auction, Dirichlet process, online learning, reserve price
\end{abstract}

\section{Introduction}

With the emergences of new wireless services and applications, the demand for spectrum is increasing. However, some studies show that many licensed spectrum bands have not been utilized efficiently [1]. In order to alleviate this contradiction, cognitive radio users, called secondary users (SUs), are proposed and allowed to opportunistically access some idle spectrum bands which belong to primary users (PUs). Before accessing idle channels, SUs should be clear about the states of primary channels. There are two main methods of obtaining idle channels: spectrum sensing and spectrum trading. In the former case, SUs do not need the cooperation of PUs and acquire spectrum opportunities only by the spectrum sensing technologies (e.g. energy detection sensing) which cost SUs' power energies and search time. In the other case, SUs who act as idle spectrum buyers provide incentives to PUs who act as idle spectrum sellers and then PUs broadcast the information of available idle channels to SUs, which is the key point in this paper.

As a result, PUs will select proper trading mechanisms to allocate idle spectrum bands to SUs for maximizing their own revenue. An auction is a very important mechanism for allocating resources efficiently in the subfield of economics and business management [2]. Hence, utilizing auction mechanisms to tackle spectrum allocation problems in

Manuscript received June 22, 2015.

Manuscript revised August 29, 2015.

Manuscript publicized November 17, 2015.

$\dagger$ The authors are with School of Electronic Engineering, University of Electronic Science and Technology of China, Chengdu, 611731 P.R. China.

${ }^{\dagger}$ The author is with School of Information and Electronics, Beijing Institute of Technology, Beijing, 100081 P.R. China.

a) E-mail: majunpaper@foxmail.com (Corresponding author)

b)E-mail: zhangyhh@uestc.edu.cn

c)E-mail: henry@bit.edu.cn

DOI: $10.1587 /$ transinf.2015EDL8140 cognitive radio networks (CRNs) is a natural idea. To our knowledge, there have been many studies on spectrum allocation problems which are based on the applications of auction mechanisms [2] in CRNs. In [3], a double auction mechanism with a reserve price is used to model the spectrum trading market with multiple sellers and buyers in which reserve prices have been used to combat the collusion behaviors among buyers. In [4], each SU can decide whether to take part in the spectrum auction based on its own rate of revenue to cost. However, previous studies do not consider how to set reserve prices to improve auctioneer's revenue without knowing the bidder's private value functions which are generally assumed to be independent probability distribution functions [5]. The proposed scheme allows the auctioneer to dynamically set reserve prices based on the historical data of the highest bids to improve its revenue during the repeated auctions.

\section{System Model}

Consider a CRN where a PU with an idle channel and $N$ SU pairs exist in the same transmission area. Both SU pairs and the PU operate in the slot transmission structure. Each SU transmitter communicates with its receiver only by the idle slots of PU's channel. The PU who acts as the auctioneer uses the second price sealed auction to sell idle slots of its channel to all SUs who act as the bidders, which can promise only one SU pair uses the idle spectrum at a time. The total auction round is $T$. In this paper, $t$ denotes the index of the auction round or the slot, which means only one auction is held in each slot. We assume the primary channel is idle during the whole repeated auction process. This assumption is rational. This is because the idle time of primary channel is much longer than the data transmission durations of SUs who have little data to transmit (like the temperature sensors). At the beginning of auction round $t$, if the channel is idle, each SU estimates the transmission rate of the idle slot by channel estimation [4], and then sends its own true estimation value as a bid to the PU. The channel rate estimated by SU pair $n$ at auction round $t$ is as follows,

$$
R_{n}^{t}=W \log _{2}\left(1+\frac{P g_{n}^{t}}{\sigma^{2}}\right),
$$

where $W$ is the channel bandwidth, $P$ is the transmit power and $\sigma^{2}$ is the thermal noise power, which are the same to all SU pairs for the simplification of research. Note that $g_{n}^{t}$ which denotes the channel gain in slot $t$ and changes over 
the time but keeps unchanged during a slot (block-fading). We also assume the PU does not know any prior information about the valuations of idle slots to the bidders and only uses the historical data to estimate the highest bid of the next auction round, and then calculates a reserve price. Here, we choose the second price-sealed auction to allocate idle slots among SU pairs because this auction mechanism is incentive compatibility which means that reporting the true channel transmission estimation value is the weak dominant strategy for all bidders [4]. This property can not be affected by setting a reserve price [5]. For this auction, SU pair $n$ with the highest bid wins the idle slot of the channel at auction round $t$, i.e.,

$$
\Omega_{n}^{t}= \begin{cases}1, & R_{n}^{t}>R_{n^{\prime}}^{t}, \forall n^{\prime} \neq n, n^{\prime}, n \in N \\ 0, & \text { else. }\end{cases}
$$

Hence, the highest bid $b_{t}^{(1)}=R_{n}^{t}$ and the secondary highest bid $b_{t}^{(2)}=\max \left(R_{n^{\prime}}^{t}, n^{\prime} \in N \backslash n\right)$ at auction round $t$. The revenue of the PU at auction round $t$ with setting a reserve price $r(t)$ is as followed,

$$
\Upsilon^{t}= \begin{cases}b_{t}^{(2)}, & b_{t}^{(2)}>r(t) \\ r(t), & b_{t}^{(1)}>r(t)>b_{t}^{(2)} \\ 0, & r(t)>b_{t}^{(1)}\end{cases}
$$

When the second highest bid is larger than the reserve price, the reserve price dose not affect the PU's revenue in this situation. Setting a reserve price is helpful to improve the revenue of PU just in the case 2 in (3) and produces a loss in the case 3 in (3). If the PU does not set a reserve price, the revenue of the PU at auction round $t$ is $b_{t}^{(2)}$, which is the scheme without reserve price.

\section{Dynamic Reserve Price}

As mentioned before, the PU does not know the private value function of each SU pair. Therefore, the available information for the PU to make a decision is only based on the history data of the highest bids and the second highest bids produced by each auction round. In our scheme, we just utilize the information of highest bids to derive the reserve price to improve the PU's revenue. In real application, the probability distribution function of the highest bids is not proper to assume that it belongs to a probability distribution family. Hence, we use Bayesian nonparametric scheme to model the sequential observed data. In the Bayesian nonparametric model, Dirichlet process (DP) [6] is a very important and useful method. In this section, we firstly introduce the DP and then the problem of how to use the DP to set reserve prices during the repeated auction process will be discussed in later subsection.

\subsection{Dirichlet Process}

Our work in this part mainly refers to [6]. Before introducing DP, we describe Dirichlet distribution because DP is a
Dirichlet distribution over infinite sample spaces. Further more, the Dirichlet distribution is the multivariate generalization of the beta distribution.

Definition 1: Let $q=\left[q_{1}, q_{2}, \ldots, q_{K}\right]$ be a distribution over the K-dimensional probability simplex, and $\alpha=$ $\left[\alpha_{1}, \alpha_{2}, \ldots, \alpha_{K}\right]$. Then, $q$ is said to have a Dirichlet distribution with parameter $\alpha$, which is denoted by $q \sim \operatorname{Dir}(\alpha)$, if

$$
p\left(q_{1}, q_{2}, \ldots, q_{K}\right)=\frac{\Gamma\left(\alpha_{0}\right)}{\prod_{i=1}^{K} \Gamma\left(\alpha_{i}\right)} \prod_{i=1}^{K} q_{i}^{\alpha_{i}-1},
$$

where $q_{i} \geq 0, \sum_{i=1}^{K} q_{i}=1$ and $\alpha_{i}>0, \alpha_{0}=\sum_{i=1}^{K} \alpha_{i}$.

Next, we introduce the DP over sample space $\Theta$. For a random distribution $G$ over $\Theta$ to be distributed according to a DP, its marginal distributions have to be Dirichlet distributed. Specifically, let $H$ be a distribution over $\Theta$ and $\beta$ be a positive real number. Then for any finite measurable partition $A_{1}, \ldots, A_{R}$ of $\Theta$ the vector $\left(G\left(A_{1}\right), \ldots, G\left(A_{R}\right)\right)$ is random since $G$ is random.

Definition 2: $G$ is DP distributed with base distribution $H$ and concentration parameter $\beta$, written as $G \sim D P(\beta, H)$, if

$$
\left(G\left(A_{1}\right), \ldots G\left(A_{R}\right)\right) \sim \operatorname{Dir}\left(\beta H\left(A_{1}\right), \ldots, \beta H\left(A_{R}\right)\right)
$$

for every finite measurable partition $A_{1}, \ldots, A_{R}$ of $\Theta$. Given observed values of $m^{1}, m^{2}, \ldots, m^{t}$, and let $n_{r}=$ $\sharp\left\{t: m^{t} \in A_{r}\right\}$, the posterior distribution on $\Theta$ is updated as

$$
\begin{aligned}
\left(G\left(A_{1}\right), \ldots G\left(A_{R}\right)\right) \mid m^{1}, \ldots, m^{t} & \\
& \sim \operatorname{Dir}\left(\beta H\left(A_{1}\right)+n_{1}, \ldots, \beta H\left(A_{R}\right)+n_{R}\right) .
\end{aligned}
$$

We are very interested in the predictive distribution of $m^{t+1}$ under condition that $m^{1}, \ldots, m^{t}$ have been given. The probability of next observation $m^{t+1}$ in a measurable $A \in \Theta$ is as follows,

$$
\begin{aligned}
& P\left(m^{t+1} \in A \mid m^{1}, \ldots, m^{t}\right)= \\
& \quad \frac{1}{\beta+t}\left(\beta H(A)+\sum_{i=1}^{t} \delta_{m^{i}}(A)\right) .
\end{aligned}
$$

For the PU, the highest bids' region which is from the minimum highest bid to the maximum highest bid can be divided uniformly into a partition $A_{1}, \ldots, A_{R}$. Furthermore, we adopt the base distribution $H$ as the uniform distribution over $\left(0, \max b_{t}^{(1)}\right)$, where $b_{t}^{(1)}$ denotes the any possible highest bid in the auction. In fact, $\max b_{t}^{(1)}$ denotes the maximal capacity of SU pairs, which is restricted by SU pairs' hardware and the channel conditions. In simulations of section IV, we assume the maximal capacity among the SU pairs is generated through all random channel conditions and is same for all SU pairs. Hence, we use $\left(0, b_{\max }^{(1)}\right)$ to denote the highest bids' region of the PU.

\subsection{Auction Procedure with Dynamic Reserve Price}

If the PU set a reserve price, it will face a situation where 
its spectrum opportunity can not be sold at some time. For example, the case 3 in (3). When the spectrum opportunity is unsold, the loss is inevitable. When auction round $t-1$ is ended, the PU can utilize the historical data of highest bid from auction round 1 to the auction round $t-1$ based on DP to set a reserve price $r(t)$. The $\hat{b}_{t}^{(1)}$ denotes the prediction of the highest bid $b_{t}^{(1)}$ at auction round $t$, and the probability of $\hat{b}_{t}^{(1)}<r(t)$ can be given,

$$
\begin{aligned}
& P_{t}\left(0<\hat{b}_{t}^{(1)}<r(t) \mid\right.\left.b_{1}^{(1)}, \ldots, b_{(t-1)}^{(1)}\right)= \\
& \frac{1}{\beta+(t-1)}\left(\beta H(r(t))+\sum_{i=1}^{(t-1)} \delta_{b_{i}^{(1)}}(r(t))\right),
\end{aligned}
$$

where $\sum_{i=1}^{(t-1)} \delta_{b_{i}^{(1)}}(r(t))$ denotes the number of highest bids producing by previous $t-1$ auction rounds which are less than $r(t)$.

If the PU set a reserve price $r(t)$, the maximal expected loss is $r(t) P_{t}\left(0<\hat{b}_{t}^{(1)}<r(t)\right)$, which is because if $0<b_{t}^{(1)}<r(t)$, the actual loss is $b_{t}^{(2)}$ which is also less than $r(t)$. When $b_{t}^{(1)}>r(t)$, the minimal expected revenue is $r(t) P_{t}\left(\hat{b}_{t}^{(1)}>r(t)\right)$, which is because the PU's revenue is $\left(b_{t}^{(2)}, b_{t}^{(2)}>r(t)\right)$ or $\left(r(t), b_{t}^{(2)}<r(t)\right)$ when $b_{t}^{(1)}>r(t)$ at auction round $t$. Hence, the additional expected revenue $y(t)$ produced by setting a reserve price $r(t)$ at auction round $t$ is as follows,

$$
\begin{aligned}
y(t) & =r(t) P_{t}\left(\hat{b}_{t}^{(1)}>r(t) \mid b_{1}^{(1)}, \ldots, b_{(t-1)}^{(1)}\right)- \\
& r(t) P_{t}\left(0<\hat{b}_{t}^{(1)}<r(t) \mid b_{1}^{(1)}, \ldots, b_{(t-1)}^{(1)}\right) \\
& =r(t)\left(1-2 P_{t}\left(0<\hat{b}_{t}^{(1)}<r(t) \mid b_{1}^{(1)}, \ldots, b_{(t-1)}^{(1)}\right),\right.
\end{aligned}
$$

where $P_{t}\left(0<\hat{b}_{t}^{(1)}<r(t) \mid b_{1}^{(1)}, \ldots, b_{(t-1)}^{(1)}\right)$ is decided by (8). From (9), if $r(t)=0$ and $y(t)=0$, which means the PU can not obtain additional revenue if it does not set a reserve price. Under this situation, the PU will receive the accumulated revenue $\sum_{t=1}^{T} b_{t}^{(2)}$ for total auction round $T$. Meanwhile, when $y(t)<0$, PU has no need to set a reserve price at the auction round $t$. We assume the PU is risk neutral, which means that the PU will pursue the maximum expected revenue during the decision-making process. When $r(t)$ increases, the $P_{t}\left(0<\hat{b}_{t}^{(1)}<r(t) \mid b_{1}^{(1)}, \ldots, b_{(t-1)}^{(1)}\right)$ also increases and $\left(1-2 P_{t}\left(0<\hat{b}_{t}^{(1)}<r(t) \mid b_{1}^{(1)}, \ldots, b_{(t-1)}^{(1)}\right)\right)$ decreases. Therefore, there must be an optimal value $r^{*}(t)$ in the area $\left(0, b_{\max }^{(1)}\right)$ to maximize $y(t)$. Therefore, We can denote the optimal value $r^{*}(t)=\operatorname{argmax}(y(t))$. Note that $r^{*}(t)$ is just the optimal value for (9). In order to find the $r^{*}(t)$, we can use the first derivative of (9). However, it is difficult to obtain the first order condition of (9) in this model. Hence, we set a search step $k$ and find $r^{*}(t)$ in the range $\left(0, b_{\text {max }}^{(1)}\right)$. The whole process of the proposed spectrum auction with setting a reserve price is described in Algorithm 1.

\section{Simulation Results}

The performance of the proposed scheme is evaluated in
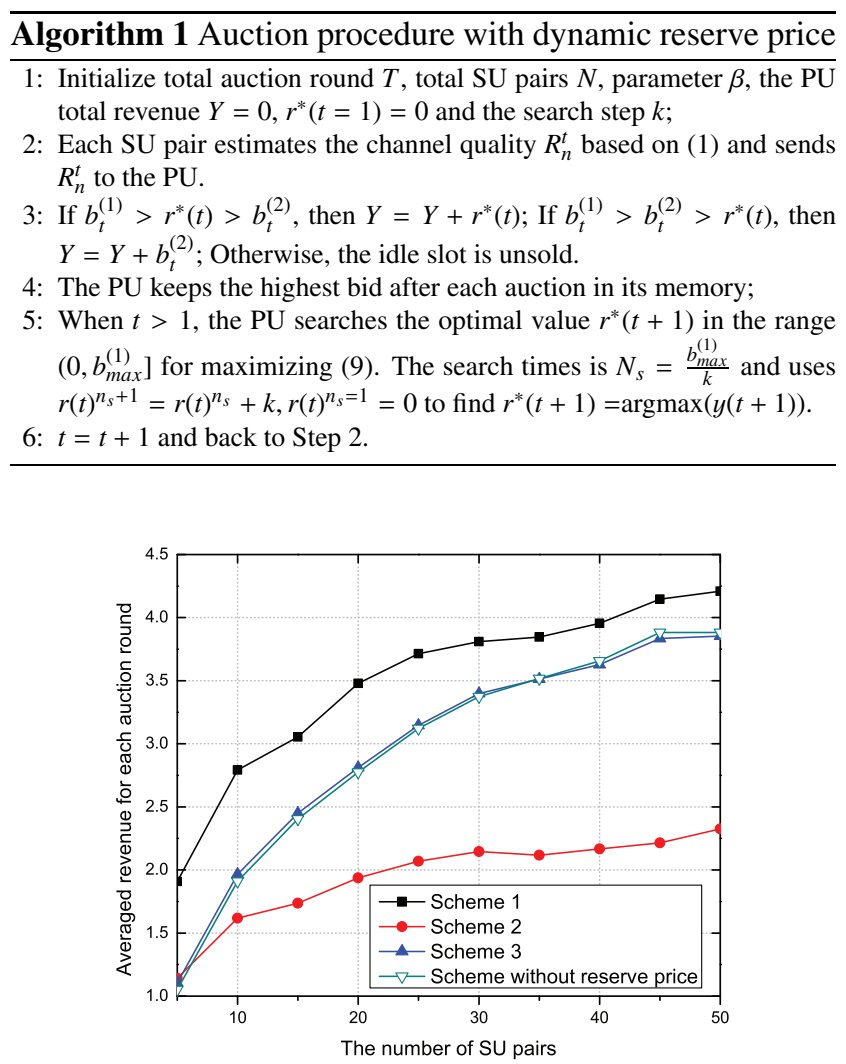

Fig. 1 Averaged revenue for each auction round

this section. There are $N$ SU pairs and one PU in a CRN covering a $500 \mathrm{~m} \times 500 \mathrm{~m}$ area. All SU pairs adopt the channel model of Flat/Light tree density proposed in [7]. The transmission power is $10^{-3} \mathrm{~W}$ and the noise power level for all SU pairs is assumed to be $10^{-12} \mathrm{~W}$. The bandwidth for all SUs is assumed as $1 \mathrm{~Hz}$, the parameter $\beta=0.5$ and the search step is $k=0.01$. In our simulations, $N=(5,15, \ldots, 50)$. When the locations of all SU transmitters and SU receivers have been selected according to the uniform distribution, the repeated auction process runs $T=5 \times 10^{3}$ times. For the averaged performance of our proposed scheme,this process will be repeated 100 times for each $N$.

In order to evaluate the performance of the proposed scheme, we compare the performance with the two other schemes. The first one is that the reserve price for each auction round is decided by the averaged value of the highest bids until auction round $t-1$, namely, $r(t)=\sum_{t^{\prime}=1}^{t-1} b_{t^{\prime}}^{(1)} /(t-1)$, which is also called Scheme 2 in the figures. The other is the the reserve price for each auction round is decided by the averaged value of the second highest bids until auction round $t-1$, namely, $r(t)=\sum_{t^{\prime}=1}^{t-1} b_{t^{\prime}}^{(2)} /(t-1)$, which is also called Scheme 3 in the figures. Firstly, we compare the PU's averaged revenue obtained by three schemes with the scheme without a reserve price during the auction process in Fig. 1. Here, Scheme 1 denotes the proposed scheme described in Algorithm 1 and the PU's averaged revenue is defined as the ratio between the total revenue obtained and the total auction 


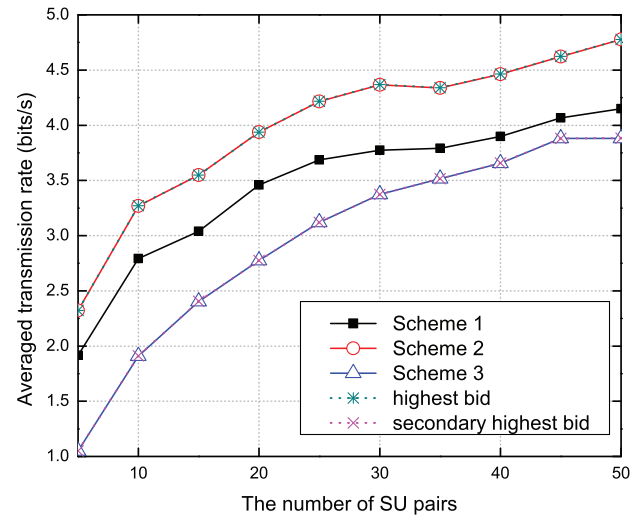

Fig. 2 Averaged reserve price for each auction round

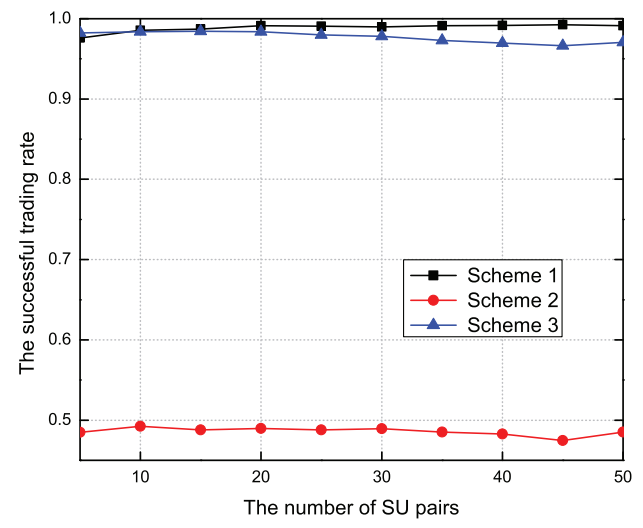

Fig. 3 Averaged successful trading rate

round. We can note that the proposed scheme outperforms another schemes in the respect of improving the averaged revenue of the PU for each auction round. The performance of Scheme 3 is close to the scheme without a reserve price. Scheme 2 is the worst case, which is because the reserve price is too high if the PU sets the reserve price according to $r(t)=\sum_{t^{\prime}=1}^{t-1} b_{t^{\prime}}^{(1)} /(t-1)$. When the reserve price is too high, too many idle slots have not been sold.

During the whole auction procedure, the reserve price is adjusted dynamically because of the updating information of the highest bid data. In Fig. 2, we demonstrate the dynamic of the averaged reserve prices as well as the averaged highest bids and secondary highest bids for each auction round. We can note that the averaged reserve price obtained by our proposed scheme is always between the averaged highest bids and the averaged second highest bids, which is helpful for the PU to improve its revenue.

In Fig. 3, We note that not all spectrum auction succeed under a predetermined reserve price. We show averaged successful trading rate (STR) between the successful trading times and the total auction round. We can note that the averaged STR by Scheme 1is more than other two cases, which means setting reserve prices by our method has a small effect on the success probability of the idle slot auction. Therefore, our method can achieve a good tradeoff between the PU's revenue and the utilization efficiency of idle

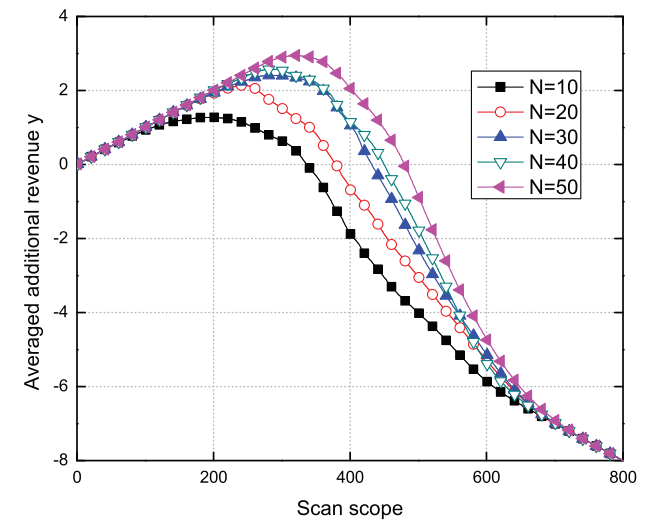

Fig. 4 Averaged additional revenue

slots.

The varied trends of averaged additional revenue during the whole auction process in Fig. 4. We can find the optimal value $r^{*}(t)$ for (9) exists because of the concavity of averaged additional revenue $y$. We also find that $r^{*}(t)$ is increasing with the increase of the number of bidders. This is because that the highest bids become larger when the number of SUs increase. Here, the search area is $\left(0, b_{\max }^{(1)}=8\right)$.

\section{Conclusion}

In this letter, we have proposed a simple method to set reserve prices to improve the PU's revenue under the condition the PU does not know the private value function of each SU pair and also does not care about the number of bidders. The PU just utilizes the limited historical information of highest bids to dynamically set a reserve price at each auction round to improve its revenue during the repeated auction procedure. Our method can also achieve a good tradeoff between the PU's revenue and the utilization efficiency of idle slots. This scheme is fit for the implementation of practical CRNs.

\section{References}

[1] B. Wang and K.J.R. Liu, "Advances in cognitive radio networks: A survey,” IEEE J. Sel. Topics Signal Process., vol.5, no.1, pp.5-23, Feb. 2011.

[2] Y. Zhang, C. Lee, D. Niyato, and P. Wang, "Auction approaches for resource allocation in wireless systems: A survey," IEEE Commun. Surveys Tuts., vol.15, no.3, pp.1020-1041, 3Q 2013.

[3] J. Zhu and K.J.R. Liu, "Multi-stage pricing game for collusion-resistant dynamic spectrum allocation," IEEE J. Sel. Areas Commun., vol.26, no.1, pp.182-191, Jan. 2008.

[4] Z. Han, R. Zheng, and H.V. Poor, "Repeated auctions with bayesian nonparametric learning for spectrum access in cognitive radio networks," IEEE Trans. Wireless Commun., vol.10, no.3, pp.890-900, March 2011.

[5] V. Krishna, Auction theory, Academic press, Waltham, 2009.

[6] Y.W. Teh, "Dirichlet process," in Encyclopedia of machine learning, pp.280-287, Springer, 2010.

[7] V. Erceg, L.J. Greenstein, S.Y. Tjandra, S.R. Parkoff, A. Gupta, B. Kulic, A.A. Julius, and R. Bianchi, "An empirically based path loss model for wireless channels in suburban environments," IEEE J. Sel. Areas Commun., vol.17, no.7, pp.1205-1211, July 1999. 